\title{
Pediatric postmortem computed tomography: initial experience at a children's hospital in the United States
}

\author{
Sharon W. Gould ${ }^{1}$ (D) M. Patricia Harty ${ }^{1}$ - Nicole E. Givler ${ }^{1} \cdot$ Theresa E. Christensen $^{2} \cdot$ Riley N. Curtin ${ }^{2}$. \\ Howard T. Harcke ${ }^{1}$
}

Received: 11 December 2018 / Revised: 17 April 2019 / Accepted: 16 May 2019 / Published online: 14 June 2019

(C) Springer-Verlag GmbH Germany, part of Springer Nature 2019

\begin{abstract}
Postmortem CT might provide valuable information in determining the cause of death and understanding disease processes, particularly when combined with traditional autopsy. Pediatric applications of postmortem imaging represent a new and rapidly growing field. We describe our experience in establishing a pediatric postmortem CT program and present a discussion of the distinct challenges in developing this type of program in the United States of America, where forensic practice varies from other countries. We give a brief overview of recent literature along with the common imaging findings on postmortem CT that can simulate antemortem pathology.
\end{abstract}

Keywords Children $\cdot$ Computed tomography $\cdot$ Forensics $\cdot$ Postmortem

\section{Introduction}

While radiographs have been used for more than a century to augment autopsy findings, ultrasound (US), CT and MRI have been introduced more recently for postmortem imaging [1-3]. Pediatric postmortem imaging is a nascent field with much to be learned regarding best practices, imaging protocols and differentiation of antemortem pathology from expected postmortem changes [1, 3-13], although interest has grown [4] with the journal Pediatric Radiology publishing a minisymposium on pediatric postmortem imaging in April 2014 based on an initiative led by the European Society of Paediatric Radiology (ESPR) [3, 7-9, 14-24]. In the USA forensic and hospital practice varies compared to other countries' legal and health systems and must be tailored to conform to local requirements. In this article we discuss the status of pediatric postmortem CT in the USA and briefly describe

Sharon W. Gould

Sharon.Gould@nemours.org

1 Department of Medical Imaging,

Nemours/Alfred I. duPont Hospital for Children,

1600 Rockland Road, Wilmington, DE 19803, USA

2 Department of Biomedical Research,

Nemours/Alfred I. duPont Hospital for Children,

Wilmington, DE, USA our hospital's experience in establishing a pediatric postmortem CT service in a particular state.

\section{Background}

Autopsy has always been considered crucial to the understanding of pathological processes and cause of death, evaluating the accuracy of clinical diagnosis, and assessing the efficacy of medical and surgical interventions. In a forensic setting, the investigation might include determining the manner of death, time of death and place of death [5], evidence for which might be crucial to judicial proceedings. Pediatric autopsies might identify underlying disorders, detect unsuspected trauma and further evaluate prenatal findings in fetuses and newborns $[7,8,11,25]$. Information gained during autopsy might be crucial for family counseling in the case of a hereditary condition $[7,8,23]$ or for criminal investigation and protection of other children at risk in the setting of nonaccidental trauma. Autopsies are important because there is a reported $10 \%$ to $25 \%$ rate of discrepancy between clinical diagnosis and full autopsy findings [8-10]. Up to $50 \%$ of medical certificates for stillborn babies might be incorrect [9]. Cardiac abnormalities are found in up to $35 \%$ of fetal autopsies, only $50 \%$ of which are detected prenatally [23]. Previously undiagnosed cardiac defects are found in up to $10 \%$ of sudden infant death syndrome (SIDS) cases [23]. 
Despite their importance, however, autopsies have significantly declined in frequency to between $5 \%$ and $30 \%$ for multiple reasons including cost and reluctance of parents to consent [1, 3, 7-9, 19, 25, 26].

Since 1895 , radiographs have been used for autopsy and forensic investigation [19]. In children, they provide an adjunct to pediatric autopsies for suspected fracture, skeletal malformation or dysplasia $[2,8,12,19,27,28]$. The overall diagnostic yield of postmortem radiographs in fetuses with no suspected skeletal abnormality is low $[2,8,12,27]$ and therefore might best be reserved for use in high-risk cases. The utility of radiographs to detect pathology might improve with higher-dose and higherresolution technique with dedicated pathological or mammographic equipment $[22,27,28]$. Skeletal surveys have been found to be useful in the setting of unexplained infant death to assess for occult fractures [19, 22, 29]. The National Association of Medical Examiners (NAME) has established minimum standards for the use of medical imaging in forensic autopsy performance [30]. Radiographs of all infants are required throughout the USA with the intent to detect occult fractures. There is no requirement beyond radiographs in this setting in the United States and technical details are not given.

Postmortem CT was introduced in 1977 for forensic use [6, 19], with the first reported use for autopsy in 1983 for identifying gas in the brain of a Navy diver [19, 31]. Postmortem CT was first suggested as a possible alternative to autopsy in 1994, although the authors described that the combination of CT and autopsy provided the most information [32]. As multidetector technology has advanced, the use of CT for postmortem evaluation in adults has steadily grown worldwide and is routine practice at some centers [19]. Multidetector CT technique has replaced single-detector technique for postmortem imaging and appears to be most helpful for assessing skeletal pathology [2, 12, 20, 33], and it might also be helpful in identifying intracranial hemorrhage [34, 35]. Numerous authors have reported applications of postmortem CT angiography to investigate vascular pathology and determine sites of large vessel hemorrhage [31,36,37], and CT angiography has been used to evaluate congenital heart disease [19]. Ventilated postmortem CT is another variation of CT technique in adults that allows for better differentiation between postmortem lung changes and underlying antemortem lung pathology [38].

Postmortem ultrasound (US) is provided at some centers in Europe, particularly for very small premature babies and fetuses $[8,12,39,40]$. Assessment of cystic hygromas or ray anomalies, such as bones along the radial aspect of the arm, wrist and hand, in these very young children might best be performed with US because these lesions can be harder to identify with $\mathrm{CT}$ [8]. In addition, US can be used to guide targeted tissue sampling to augment postmortem imaging findings [8, 40]. Postmortem MRI has proved valuable in assessing the brain and spinal cord in children, even very young fetuses $[8,9,12,41]$. In the setting of more advanced brain decomposition, postmortem MRI might provide the opportunity to evaluate brain tissue even when fixation for histological evaluation is no longer possible [11, 42]. MRI has been shown to be highly reliable in the postmortem evaluation of very young children and fetuses when combined with other noninvasive techniques such as laboratory tests and external examination $[3,4,11,43]$. Postmortem MRI has been shown to be superior to postmortem CT for the evaluation of soft tissues [41], particularly the central nervous system $[8,12]$ and heart [23].

Publications on the topic of postmortem imaging continue to increase $[4,6]$, including the creation of a dedicated forensic imaging journal, the Journal of Forensic Radiology and Imaging, and terminology and best practices are being developed through the creation of international task forces in Europe [12, 19, 25] and North America [44]. In 2014 Arthurs et al. [2] conducted a survey of ESPR members regarding postmortem imaging practices. They reported that $71 \%$ of respondents perform some form of pediatric postmortem imaging. Almost half of institutions performed imaging in some stillbirths, neonatal deaths and infant deaths. Medical examiner referrals constituted $13 \%$ of cases, and only one center imaged all autopsy cases [2]. Eighty-one percent of institutions offered radiographs, $51 \%$ performed postmortem CT and 38\% used postmortem MRI. Eighty-seven percent of imaging was performed in radiology departments exclusively, with most imaging performed by clinical radiologic technologists [2]. Most institutions providing postmortem CT had a standard protocol, often a whole-body technique, although some centers used a separate head protocol. Pediatric radiologists most commonly interpreted the studies, sometimes with pathologists' input [2]. In 2016 the SPR Postmortem Imaging Task Force reported a survey conducted by Harty and Schmit [44] of the Society of Chiefs of Radiology at Children's Hospitals (SCORCH) members regarding postmortem imaging practices in North America. Of the responding departments, $60 \%$ offered perinatal postmortem imaging and 55\% offered forensic imaging. Most departments saw fewer than 20 cases per year. All offered radiographs, with most offering $\mathrm{CT}$ for forensic cases, a smaller percentage utilizing MRI or CT for perinatal death imaging, and $31 \%$ performing forensic MRI. Most centers did have access to pathology reports in either type of case, and most did not receive any form of reimbursement for either type of case. Staff radiologists reported postmortem cases in all departments, although most departments did not have designated postmortem readers. Most institutions that performed fetal postmortem imaging had set radiography and CT protocols, but only half had set MRI protocols. Of institutions that performed forensic postmortem imaging, most had set protocols for all modalities [44].

To date, interest and experience in postmortem CT in the USA have lagged behind Europe in both hospital and medicolegal death investigation. There is no reimbursement 
schedule for postmortem CT by insurance carriers and coroner/medical examiner jurisdictions are underfunded and reluctant to order studies if they are charged. A major challenge to instituting a postmortem imaging program, therefore, is to win the support of hospital administration and staff to provide an adjunct or alternative to conventional autopsy that they are willing to offer both families and the medicolegal community for medicolegal investigations for which there will be no reimbursement. In addition, the United States configuration of 50 states with independent statuses as well as differing state and local regulations relating to medicolegal death investigation results in the need to consider local requirements when instituting a postmortem $\mathrm{CT}$ program that offers services to state government agencies.

\section{Clinical applications of postmortem CT}

In the case of stillbirth, abortion or neonatal death, imaging might help to corroborate prenatal findings or detect unsuspected underlying disease $[2,8,9]$. The knowledge gained from postmortem evaluation is helpful with parental bereavement as well as with genetic counseling [3, 8]. In the setting of skeletal dysplasia, postmortem CT can assess the phenotype with more specificity than prenatal imaging $[8,22]$ and might be more useful than radiographs except for evaluation of the fingers and toes [19], although further corroboration is needed. Postmortem CT can be used for skeletal and lung assessment in fetal/neonatal death, but Arthurs et al. [41] have found that because of poor soft-tissue contrast $\mathrm{CT}$ is less reliable than MRI, particularly in fetuses, unless specific skeletal evaluation is required. Postmortem CT has been used to identify small amounts of air in the airway and lungs in a neonate to help determine whether the child was alive at birth versus being stillborn [26, 45] (Fig. 1); however, radiographs or MRI [46] could be utilized for this purpose, as well.

In the setting of unexplained infant death, postmortem CT can be valuable for detecting intracranial hemorrhage or fractures that indicate non-accidental trauma $[12,19$, 33-35, 47] (Figs. 2 and 3). CT has been shown to be more sensitive than radiographs for assessing rib fractures [19, 47, 48], although radiographs might be more sensitive for phalangeal fractures [19] and metaphyseal corner fractures (classic metaphyseal lesions); more study is needed comparing CT to radiography in this setting [13]. In the absence of signs of trauma, however, finding the cause of death is more challenging because expected postmortem changes and antemortem lung and brain pathology can have a similar appearance on postmortem CT $[20,34,35$, 43, 49]. One study performed in France in 2013 evaluated

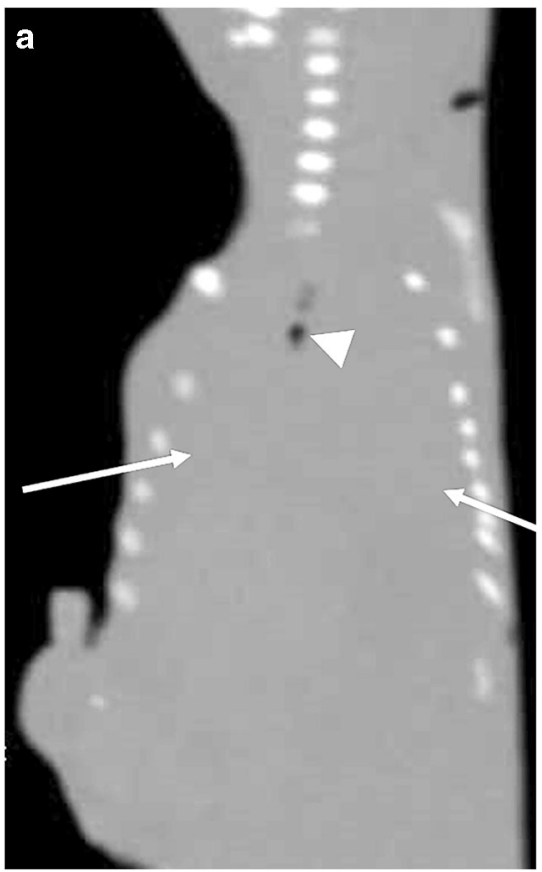

Fig. 1 Investigating apparent stillbirth. a Oblique coronal reformatted postmortem CT image of a 22 -week fetus displayed with a bone window reveals no gas in the lungs (arrows), likely because the fetus was truly stillborn. The small amount of gas centrally (arrowhead) might be in the trachea or esophagus, possibly related to resuscitative efforts or decomposition. b Coronal reformatted CT image of a 24week fetus displayed with a bone window demonstrates gas in the

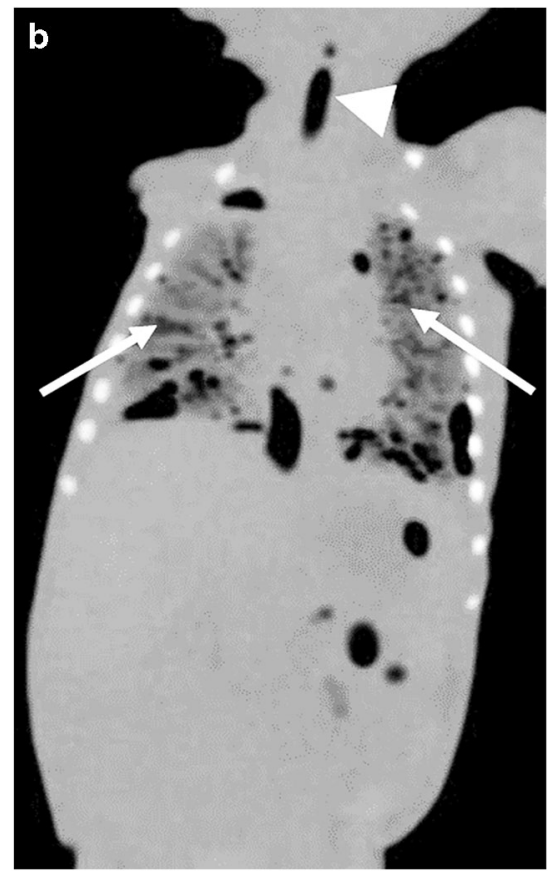

lungs (arrows) and trachea (arrowhead), a finding that suggests the infant inhaled at birth. Infants born in water might aspirate fluid rather than air, however, and so gas would not be found in the lungs and airway. Correlation with information about the birth, if known, and with assessment of endotracheal fluid and histopathology is needed. The autopsy reports for these cases were unavailable 

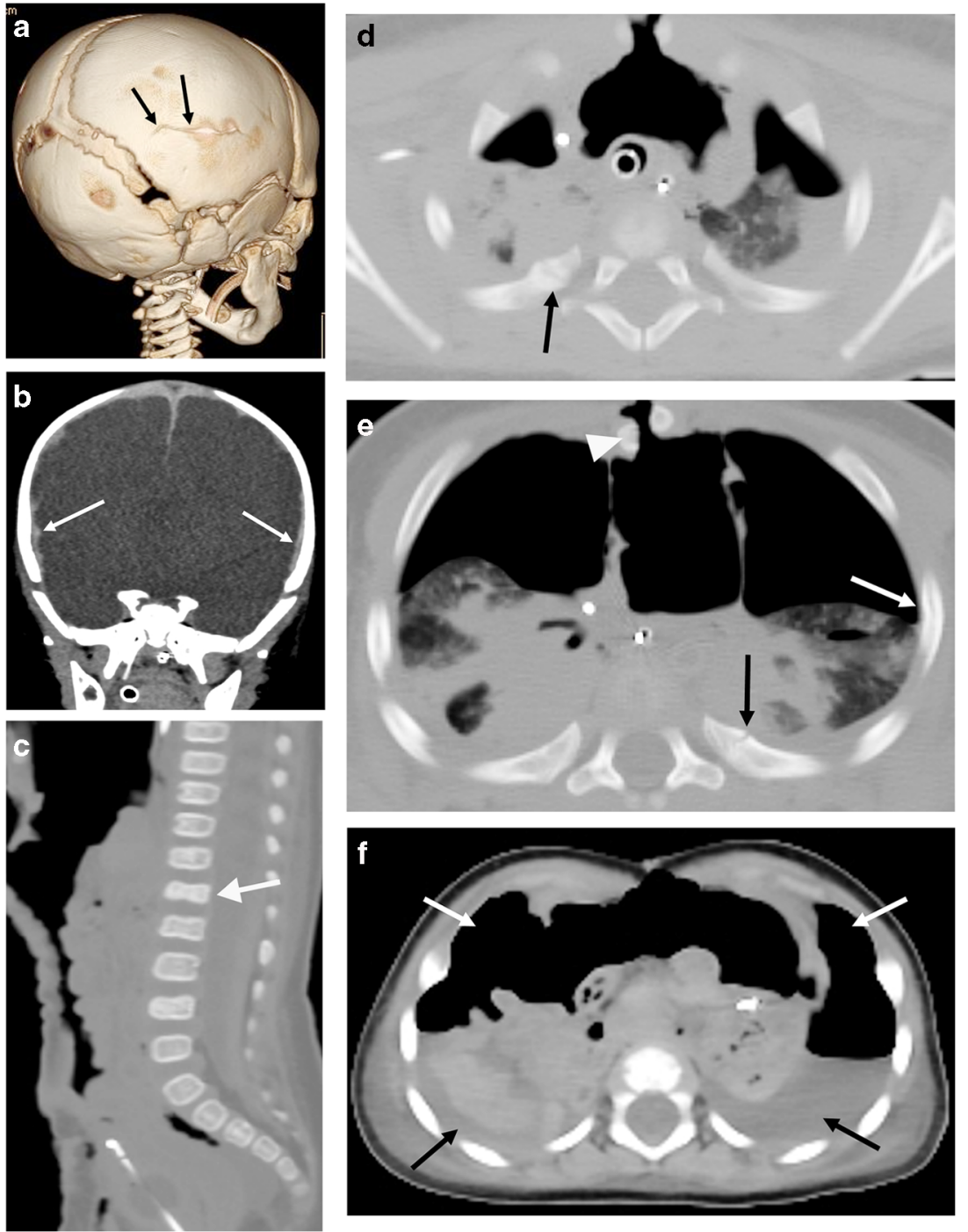

Fig. 2 Non-accidental trauma investigation in a 3-month-old boy who had recent hospital admission for pneumomediastinum, sepsis and meningitis. Rib fractures were found on a chest radiograph, raising concern for non-accidental trauma. The infant subsequently developed brain edema and was declared brain-dead. The medical examiner requested a postmortem $\mathrm{CT}$ that was performed following organ harvest. a A transverse non-depressed right parietal skull fracture (arrows) is well evident on 3-D volume-rendered CT image of the skull viewed from the right. b Coronal reformatted CT image shows diffuse loss of gray-white differentiation and bilateral temporoparietal subdural collections (arrows). c Sagittal reformatted CT image through the spine shows compression fractures of L1, L2 and L4 with mild retropulsion of

47 cases of sudden infant death and found that autopsy determined the cause of death in $38 \%$ (18 cases) [34]. CT was in concordance with autopsy in all but 3 of the 18 cases, with all discrepancies related to lung evaluation in pneumonia. CT correctly identified 4 cases of nonaccidental trauma in a cohort of children primarily younger than 2 years (mean 6 months). In all but two unexplained cases of death, no pathological CT findings were identified, with the two false-positive CT scans interpreted as
L1 (arrow). d Axial CT image through the chest shows healing right posterior rib fracture with callus (arrow). e Axial CT image through the chest shows healing left posterior 6th rib fracture (black arrow) and acute lateral 4th rib fracture (white arrow). Sequelae of organ harvest are apparent with split sternum (arrowhead), intrathoracic gas, collapsed lungs and absence of the heart. f Axial CT image at T12-L1 demonstrates the sequelae of organ harvest in the abdomen with intracavitary gas (white arrows), intracavitary fluid (black arrows) and absence of the liver and kidneys. Manner of death was determined to be "homicide"; cause of death was determined to be "shaken baby, blunt force trauma to the head." CT identified the brain edema, extra-axial collections, right parietal fracture and multiple rib fractures

pneumonia [34]. Another study in Japan involving a slightly older cohort (mean age 1.6 years) in whom nonaccidental trauma had already been excluded on the basis of clinical investigation found that CT alone infrequently determined the cause of death. However in concert with history, physical examination and laboratory values the cause of death could be identified when this information was combined with postmortem CT findings [50]. Postmortem CT has been shown to be superior to autopsy 

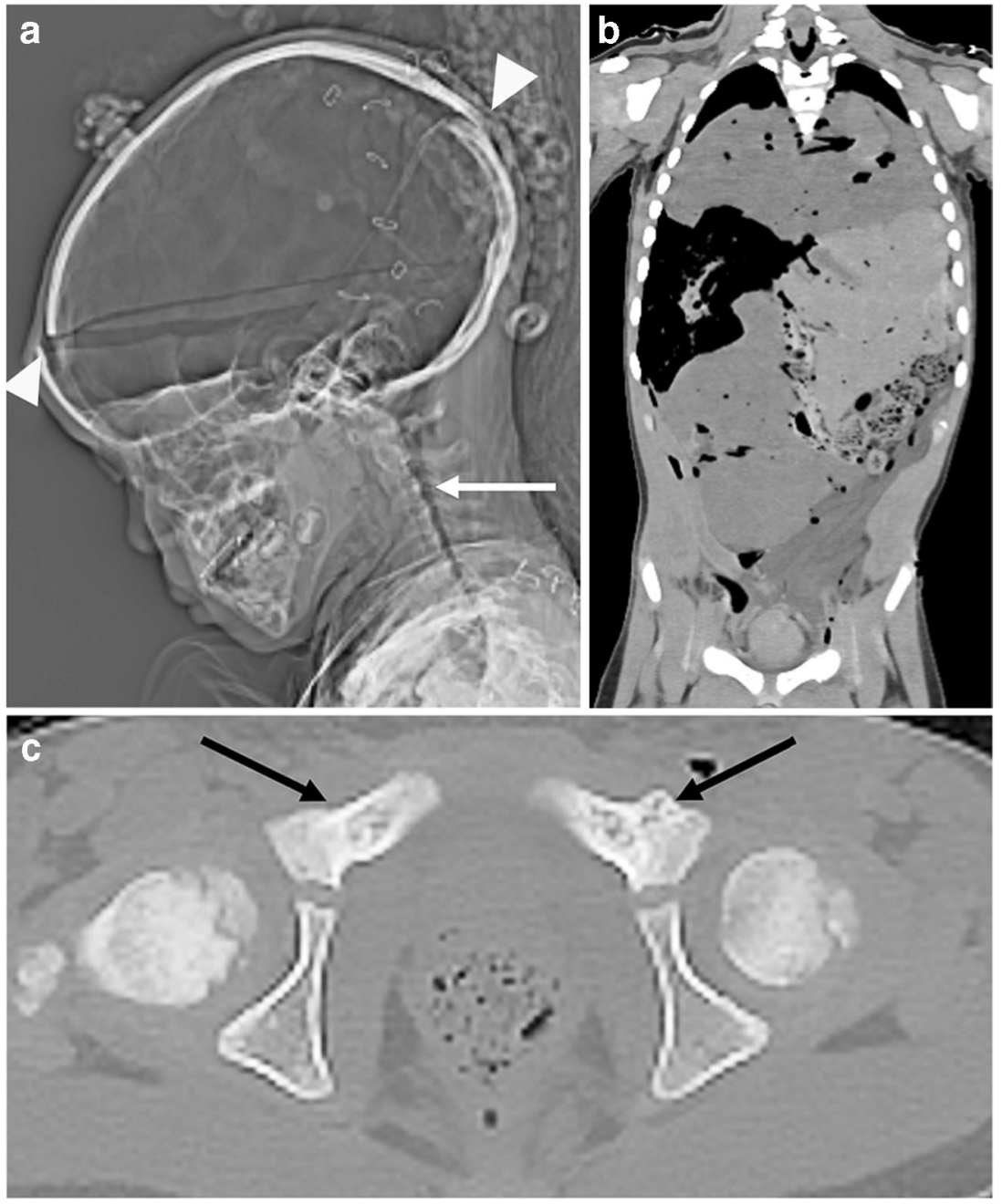

Fig. 3 Non-accidental trauma investigation in a 3-year-old girl who was found unresponsive after a brief illness that included nausea and vomiting. There was no initial clinical suspicion for abuse and the body was buried. Additional information became available and further investigation raised suspicion for abuse. The body was exhumed 2 days after burial and underwent autopsy and postmortem $\mathrm{CT}$, as requested by the medical examiner. Autopsy revealed evidence of blunt abdominal and pelvic trauma but reported no fractures. a Lateral scout image for the CT performed after autopsy shows defects in the frontal and occipital bones from craniotomy (arrowheads), with gas filling the calvarium and cervical spinal canal (arrow) following removal of the brain and cervical cord. b Coronal reformatted CT image through the trunk shows displacement of thoracic and abdominal contents after the organs were replaced following autopsy. c Axial CT image through the pelvis shows irregular contour of both superior pubic rami, compatible with healing fractures (arrows). Vertical fissures that are occasionally seen in the pubic bones of infants should be fused by approximately 1 year of age. Manner of death was determined to be "homicide"; cause of death was determined to be "blunt force to the abdomen." Postmortem CT identified pelvic fractures that were considered to be consistent with the history of pelvic trauma for detecting fractures and therefore might be most useful in cases of unnatural death with known or suspected trauma $[33,51,52]$.

Postmortem CT can also be used with older children for assessing occult trauma or occult diagnoses in medically complex children with sudden death (Fig. 4). Postmortem $\mathrm{CT}$ provides a noninvasive evaluation of placement of support devices for feedback and performance improvement and might also detect complications and clinically unrecognized conditions (Fig. 5). Detection of complications of medical or surgical interventions with postmortem $\mathrm{CT}$ has been reported as well $[36,53]$. Some authors suggest that postmortem CT helps determine the time of death [5].
Postmortem MRI has been shown to correlate well with autopsy findings in infants and children, particularly for the central nervous system, the heart and the kidneys [43]. CT performs less well than MRI overall when compared in postmortem imaging of fetuses and children as a group [41].

\section{Technical and strategic considerations}

Flach et al. [6] described in detail their postmortem CT scanning technique in 2014, and more recently Shelmerdine et al. [13] reported CT technique guidelines developed through a 

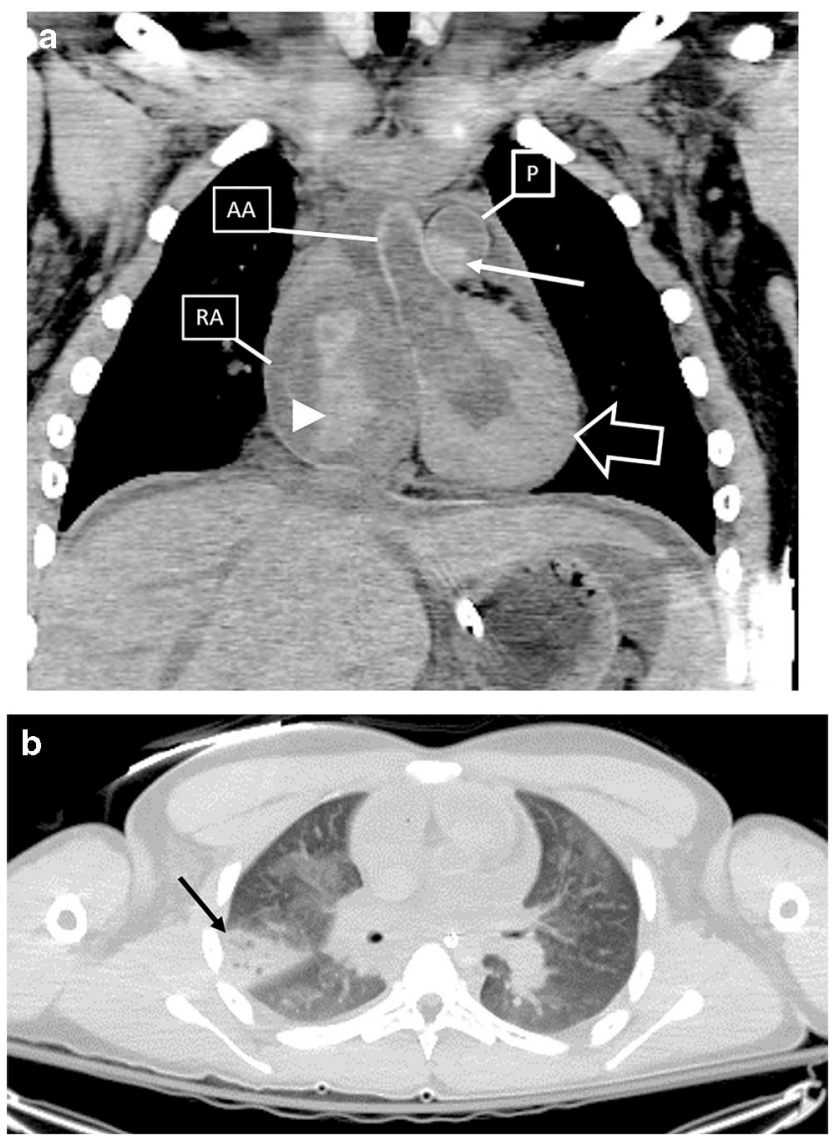

Fig. 4 Sudden death investigation in a 15-year-old boy who died in the intensive care unit following cardiac arrest and seizures after experiencing a sudden flare of nephrotic syndrome. The interval between death and imaging was $4 \mathrm{~h}$. a This coronal reformatted CT image with subdural windowing through the mediastinum shows a central hyperdense saddle pulmonary embolism (thin arrow) in the main pulmonary artery $(P)$. The embolus extended into the right and left pulmonary arteries. A hyperdense filling defect (arrowhead) in the right atrium $(R A)$ likely represents additional thrombus. The wall of the ascending aorta $(A A)$ is hyperdense and thickened and the left ventricular myocardium (thick arrow) appears thickened, expected postmortem changes that were likely related to muscle contraction. b Axial CT image taken just below the main pulmonary artery in the chest and windowed for the lungs shows an asymmetrical wedge-shape opacity indicating a pulmonary infarct (arrow). Manner of death was determined to be "natural" and cause of death was determined to be "pulmonary embolus." Blood coagulation from homeostasis tends to lie dependently in the heart and vessels. The central filling defect in the pulmonary arteries along with the asymmetry of the pulmonary parenchymal finding raises suspicion for antemortem pathology and can help direct the autopsy

joint effort of the ESPR and the International Society for Forensic Radiology and Imaging (ISFRI) and based on current practices of a large number of surveyed institutions. Goals of postmortem CT technique include very-high-resolution imaging allowing for multiplanar reformatting and threedimensional (3-D) rendering as well as detection of subtle pathology such as fractures. Because radiation exposure is not a concern, a higher-dose, higher-resolution technique is recommended to reduce image noise and reduce the likelihood of streak artifact from remaining support apparatus [54]. We

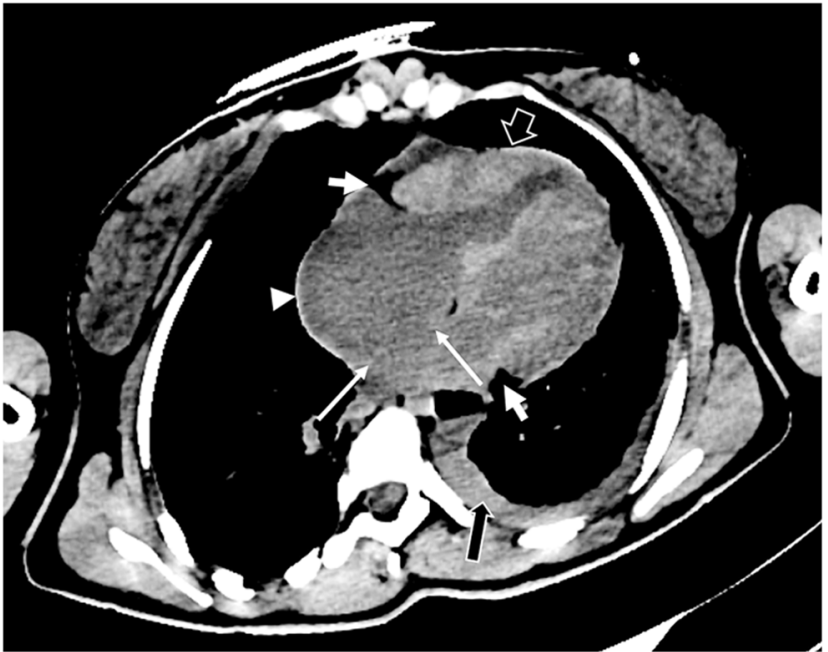

Fig. 5 Investigating sudden death in a teenage girl who was a trauma victim. Imaging was performed $2-3 \mathrm{~h}$ postmortem. This oblique axial CT image oriented to the long axis of the heart shows a very large atrial septal defect. Long white arrows indicate the expected location of the atrial septum. Short white arrows indicate fat at the atrioventricular groove, a normal finding. Right ventricular wall thickening (short black arrow) might indicate pathology associated with atrial septal defect (ASD) but myocardial thickening might also be caused by muscular contraction, an expected postmortem change. Right atrial dilatation (arrowhead) might also be secondary to ASD but can be seen with blood stasis and pooling. Autopsy revealed cardiomegaly, right ventricular dilation and hypertrophy with an abnormal, thickened tricuspid valve. The left pleural effusion (long black arrow) might have been present prior to death because bilateral chest tubes (not shown) had been removed before the decision was made to perform CT. Support catheters and lines should always be left in place for postmortem evaluation. Postmortem CT identified the atrial septal defect but could not differentiate postmortem change from true chamber dilatation and ventricular hypertrophy. The CT demonstrated no other abnormalities and excluded fractures. Pulmonary hypertension was considered a contributing factor in the cause of death. Further clinical information is not provided out of concern for patient confidentiality

present our modified multidetector technique for pediatric cases in Table 1 along with techniques described by Flach et al. [6]. As indicated in the table, we use both the smaller 16-cm field-of-view setting available on CT scanners in the USA for the brain, neck, and spine for infants as well as the larger 32$\mathrm{cm}$ field-of-view setting to image the entire body so that the field of view is as tailored to the size of the child as possible. As described by Shelmerdine et al. [13], we do not use dose modulation. While multiple $\mathrm{CT}$ runs add time to the examination, we have found the improved resolution is desirable. We perform extensive multiplanar reconstructions and 3-D volume renderings of each extremity segment, the skull, the spine, and the ribs and pelvis (Fig. 6). We find that the detailed anatomy provided by the axial raw data, multiplanar reformatted images, as well as the 3-D renderings allows for thorough assessment of skeletal structures. The recently published ESPR and ISFRI pediatric postmortem CT guidelines recommend a simpler technique utilizing a single scan from head to toe with a pitch of $<1$, submillimeter collimation and a 
Table 1 Postmortem CT technique

\begin{tabular}{|c|c|c|c|c|c|c|c|c|c|c|}
\hline \multirow{2}{*}{$\begin{array}{l}\text { Scanner } \\
\text { Study }\end{array}$} & \multicolumn{10}{|c|}{$\begin{array}{l}\text { SOMATOM Flash Definition (Siemens, Forchheim, Germany) } \\
2 \times 128 \text { slices, dual-source scanner }\end{array}$} \\
\hline & \multirow[b]{2}{*}{ Age } & \multicolumn{4}{|c|}{ Flach et al. [6] } & \multicolumn{5}{|c|}{ Authors' hospital $^{\mathrm{a}}$} \\
\hline Body part & & $\mathrm{kV}$ & Ref mAs & Pitch & $\mathrm{eFOV}(\mathrm{mm})$ & $\mathrm{kV}$ & Eff mAs & Ref. mAs & Pitch & $\mathrm{FOV}(\mathrm{mm})$ \\
\hline \multirow[t]{4}{*}{ Whole body ${ }^{\mathrm{b}}$} & $\begin{array}{c}\text { General }(5+\text { years } \\
\text { at our hospital) }\end{array}$ & 120 & 400 & 0.35 & Up to $780^{\mathrm{c}}$ & DE $80 / 140$ & & 290 & 0.7 & 320 \\
\hline & 9 mos. -5 years & & & & & 120 & 480 & & 0.7 & 320 \\
\hline & $<9$ mos., ears to toes & & & & & 120 & 480 & & 0.4 & 320 \\
\hline & $>9$ mos., head to pelvis & & & & & 120 & 110 & & 0.6 & 160 \\
\hline \multirow[t]{3}{*}{ Head and neck ${ }^{\mathrm{d}}$} & $\begin{array}{l}\text { General }(5+\text { years } \\
\text { at our hospital })\end{array}$ & 120 & 80 & 0.7 & $\mathrm{Up}$ to $300^{\mathrm{c}}$ & DE $80 / 140$ & & 290 & 0.7 & 160 \\
\hline & 9 mos. -5 years & & & & & DE $80 / 140$ & & 264 & 0.7 & 160 \\
\hline & $<9$ mos. & & & & & 120 & 110 & & 0.6 & 160 \\
\hline Thorax and abdomen & General & 120 & 400 & 0.6 & Up to $500^{\mathrm{c}}$ & & & & & \\
\hline \multirow[t]{4}{*}{ Extremities $^{\mathrm{e}}$} & $5+$ years, pelvis to toes & & & & & 140 & 400 & & 0.4 & 320 \\
\hline & 9 mos. -5 years, pelvis to toes & & & & & 120 & 480 & & 0.4 & 320 \\
\hline & $\begin{array}{l}<9 \text { mos., each upper } \\
\text { extremity separately }\end{array}$ & & & & & 120 & 480 & & 0.4 & 320 \\
\hline & $\begin{array}{l}<9 \text { mos., lower } \\
\text { extremities together }\end{array}$ & & & & & 120 & 480 & & 0.4 & 320 \\
\hline
\end{tabular}

$D E$ dual energy, Eff $m A s$ effective milliampere-seconds (unique to dual-energy scanning), $e F O V$ effective field of view, $F O V$ field of view, $k V$ kilovoltage peak, mos. months, Ref $m A s$ reference milliampere-seconds

${ }^{a}$ At our institution, raw data are obtained for all scans at $0.6 \mathrm{~mm}$ with varying overlap as indicated by pitch $<1$. For interpretation, axial brain/neck reconstructions are then made at $5 \mathrm{~mm}(3 \mathrm{~mm}$ if $<1$ year old $)$ with a soft-tissue algorithm and separately with a bone algorithm. Coronal and sagittal reconstructions are made similarly. Three-dimensional volume-rendered images of the skull are generated. For the torso, soft-tissue algorithm and bone algorithm reconstructions are generated in the axial plane at $5 \mathrm{~mm}$ ( $3 \mathrm{~mm}$ for infants). The axial series of the torso includes the lower extremities scanned together in most cases. Alternative strategies might be needed for very large patients or rigor mortis. Sagittal and coronal images through the torso only are generated at 5-mm thick (3-mm for infants) with soft-tissue and bone algorithms, as well. Two-millimeter axial lung algorithm images are also generated through the thorax. Volume-rendered 3-D images of the ribs and thoracic spine, the whole spinal column, the whole torso including pelvic and shoulder girdles, and individually of each lower extremity are made. Additional coronal and sagittal bone algorithm reconstructions are made along the long axis of each thigh and lower leg/foot segment individually at $5 \mathrm{~mm}$ (3 $\mathrm{mm}$ for infants). Separate scans of each upper extremity are performed in most cases with 5-mm soft-tissue and bone algorithm reconstructions (3- $\mathrm{mm}$ for infants). Individual coronal and sagittal bone algorithm reconstructions are made at $5 \mathrm{~mm}$ of each upper arm and forearm/hand segment as well as 3-D volume-rendered images of each upper extremity ( $3 \mathrm{~mm}$ for infants). One raw data $0.6-\mathrm{mm}$ set in the soft-tissue algorithm of each head-neck/torso/extremity scan is sent to the archiving system as well in case the radiologist needs additional reconstructions

${ }^{\mathrm{b}}$ Whole-body scans for Flach et al. [6] were truly head to toe, whereas our whole-body scans included ears to toes for children younger than 5 years and ears to pelvis for children 5 years and older. Upper extremities are included in these scans in all ages except infants younger than 9 months

${ }^{\mathrm{c}}$ The authors state that the field of view should be tailored to body habitus but can be extended up to the parameters listed

${ }^{\mathrm{d}}$ Head and neck imaging for infants is extended to include a small-field-of-view scan of the entire spine at our hospital

${ }^{\mathrm{e}}$ Because of the high percentage of cases for which we are asked to assess skeletal structures for signs of non-accidental trauma, we have instituted dedicated extremity scans for higher-resolution imaging of the immature bones

field of view tailored to patient size with no dose modulation [13]. Reconstructions are performed with soft-tissue and bone algorithms for the whole body as well as a brain algorithm for the head and a lung algorithm for the thorax. Multiplanar and 3 -D volumetric reconstructions are also included. The authors advocated high-quality radiographs for evaluating the extremities rather than reconstructions because of concern for the conspicuity of metaphyseal corner fractures on CT, although the authors stated that further study is needed to compare the utility of radiography versus CT for extremity evaluation [13].

Computed tomography scanning technique is only one facet of the technical considerations distinct to postmortem imaging.
Requests for postmortem studies arise from the clinical service in the case of an inpatient, from the emergency department in the case of trauma or sudden infant death, from genetics in the case of a known or suspected fetal abnormality, or from the medical examiner. Consent from the family is required for postmortem imaging just as it is for autopsy. The exception in the USA is when the coroner/medical examiner takes jurisdiction, he or she can mandate imaging and autopsy without parental consent. Typical medical examiner cases are suspected homicides, accidents, unwitnessed death and death occurring within $24 \mathrm{~h}$ of hospital admission. Timing of the scans is subject to decisions regarding 24-h/7-day per week scanner availability 

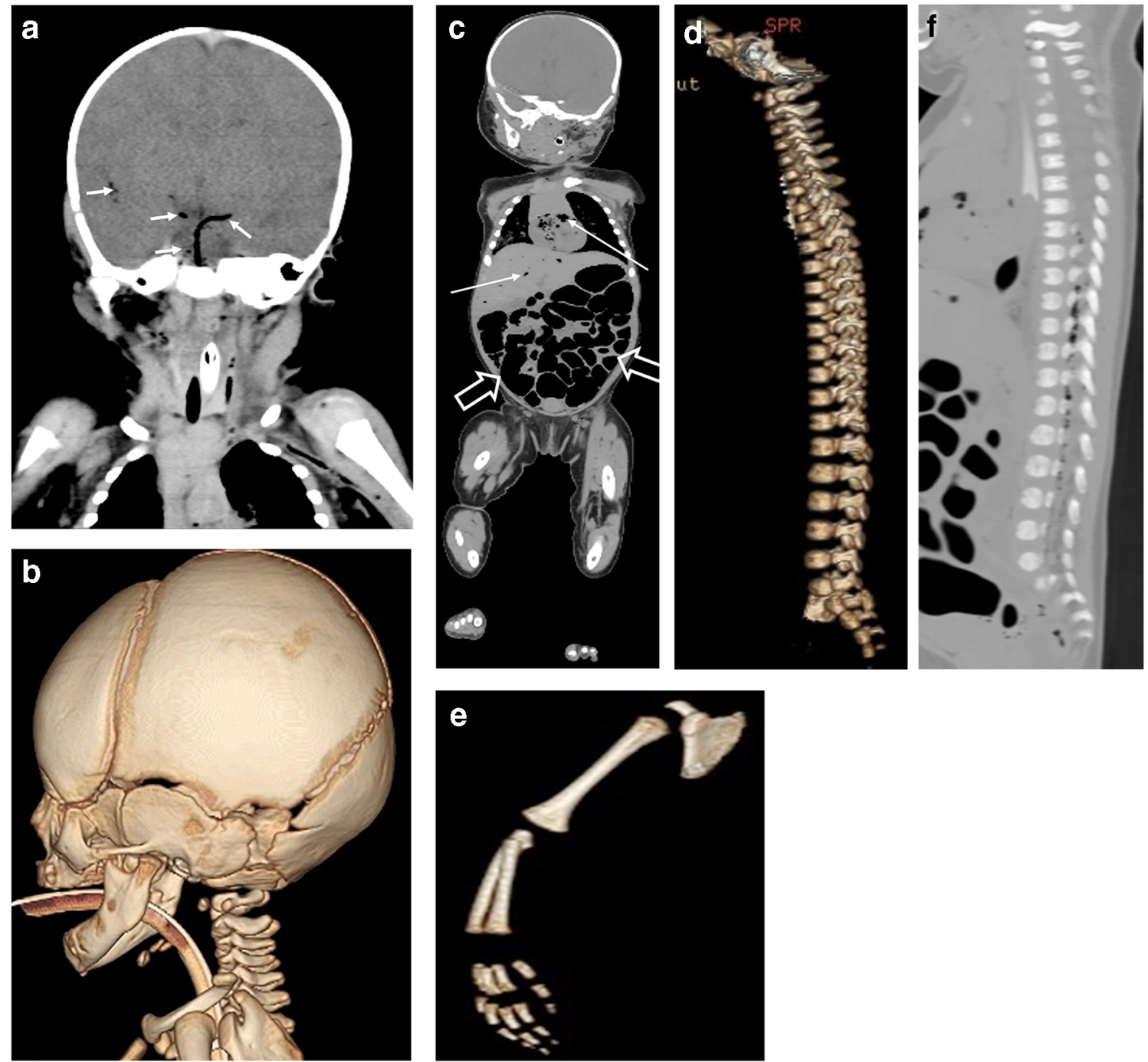

Fig. 6 Investigation in a 4-week-old girl who was referred by the medical examiner for unexplained death. Co-sleeping was suspected because no evidence of trauma was identified. The time of death was unknown. a Coronal reformatted CT image of the brain shows intravascular and parenchymal gas (arrows) that represents early putrefaction, which is an expected postmortem finding. The $16-\mathrm{cm}$ field of view is recommended for optimal detail for evaluating the brain and cervical spine and can also be used to image the entire body in very small infants. b A 3-D volumerendered $\mathrm{CT}$ image of the skull is generated to maximize the chance of detecting a subtle skull fracture. $\mathbf{c}$ A pitch of less than 1 is used to scan the entire body. Reconstructions are performed with overlapping 0.6-mm slices for optimal image quality and reduced artifact like in this coronal image. Reconstruction kernels can be chosen to optimize assessment of

lungs, bones and soft tissues. Gas identified in the liver and heart (thin arrows) represents postmortem change. Mild bowel distension with gas is also noted (thick arrows), also representing anticipated postmortem change. Three-dimensional volume-rendered CT images of the entire skeleton are made in segments for improved assessment of trauma: (d) spine and (e) right upper extremity. Multiplanar reformatted images are also created for each bony segment: (f) Sagittal reformat of the thoracolumbar spine shows normal alignment, no apparent fracture and paraspinal intravascular gas, a postmortem change. There were no fractures in this case. CT findings correlated with autopsy and found no evidence of trauma. Manner of death was "undetermined" and cause of death was signed out as "sudden unexplained infant death"

and transport of the body [7]. With forensic cases autopsy is expected within $24 \mathrm{~h}$ and postmortem CT likely involves transportation of the body with requirements for chain of custody. Consequently limited time is available and a study must be integrated with the clinical schedule. Discreet transport of the body through patient areas must be considered as well. Technologist support should be offered [7] in terms of training as well as counseling if needed. Although the postmortem CT is fairly simple to obtain because there are set techniques and there

are no concerns about radiation exposure or motion artifact, the reconstructions are time-consuming.

Image interpretation is another area requiring planning [7]. At most institutions, pediatric radiologists interpret these examinations because of their knowledge of both pediatric and fetal pathology and imaging $[2,8,9,13]$. However, because postmortem findings can mimic pathology, close collaboration with pathology or the medical examiner or both is required $[7,11,19,26]$. In addition, in 
cases involving fetal or neonatal death, collaboration with genetics and neonatology is recommended to optimize interpretation of the findings $[8,14,18]$. Another factor is the timing of image interpretation [7]. What happens with cases that are imaged during off-hours or weekends? Do the studies wait until more pressing cases involving living patients have been completed? Details regarding the timing and extent of the postmortem imaging services to be offered must be decided.

\section{Interpretation of findings}

Expected processes ensue during and after death that affect tissue appearance on postmortem imaging and these must be taken into consideration. Familiarity with the imaging appearance of these processes is necessary for accurate interpretation of postmortem imaging [9, 18, 55-57]. Livor mortis, rigor mortis and decomposition processes such as autolysis, maceration and putrefaction all alter the appearance of affected tissue when compared with in vivo imaging [20, 21, 49]. A complete discussion of postmortem processes is beyond the scope of this article, but excellent descriptions can be found in the papers by Klein et al. [20], Arthurs et al. [21] and Offiah and Dean [49]. A critical point to consider is that the length of the postmortem interval, the time interval between the actual death of the child and imaging, the temperature and humidity at which the remains have been kept, and any disease processes such as fever can alter the rate at which postmortem changes occur [20, 21, 49]. For this reason, the extent of postmortem change, for example the amount or distribution of gas in the soft tissues or the extent of autolysis, does not correlate with postmortem interval [34]. One group has found some degree of reliability in establishing time of death by measuring the Hounsfield units (HU) of the cerebrospinal fluid in adults, but further corroboration in children is needed [5].

Within the brain, autolysis begins soon after death and decreases the differentiation between gray and white matter, simulating brain edema [21, 49, 58] (Fig. 7). The changes in gray-white matter differentiation are accompanied by swelling of the brain and descent of the cerebellar tonsils [21, 49, 58-60]. Distinguishing between antemortem brain edema and postmortem autolysis can be very difficult [34, 43], although Berger et al. [58] suggested that narrowing of the temporal horns might be a reliable sign of antemortem onset of edema. Detection of intracranial hemorrhage is an important finding $[31,34]$. Hemorrhage is never an expected result of postmortem change [56] (Fig. 8). Postmortem CT might not detect very small amounts of extra-axial subdural or subarachnoid hemorrhage but is more sensitive than autopsy for small amounts of interhemispheric blood [61].

Several changes occur within the cardiovascular system that can be confused with antemortem disease. Because of hemostasis, solid components of the blood settle dependently, creating a visible hematocrit effect with visible intravascular and intracardiac fluid/fluid levels [20, 21, 34, 49, 56, 57, 59] (Fig. 9). The higher attenuation solid blood components lie dependently within the vascular lumen. Higher attenuation material in the center of the lumen should raise the question of antemortem thrombus [49, 59] (Fig. 4); however

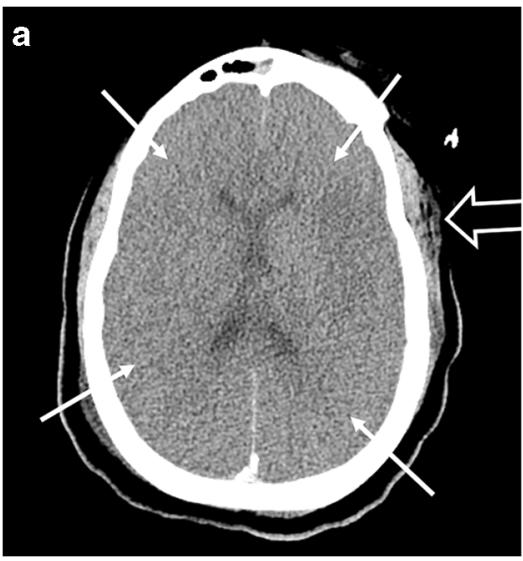

Fig. 7 Differentiating normal postmortem processes from pathology in a 16-year-old girl who was admitted to another hospital after being found unresponsive in a group home where she lived because of a combination of developmental intellectual disability and multiple psychological diagnoses. Her admitting diagnosis was anoxic brain injury from cardiac arrest. She died in the hospital after another episode of cardiac arrest. Postmortem CT was requested by the medical examiner. There was an interval of $6 \mathrm{~h}$ between the time of death and the CT. a There is diffuse loss of gray-white matter differentiation (thin arrows) on this unenhanced axial CT image. This finding could represent autolysis, an expected

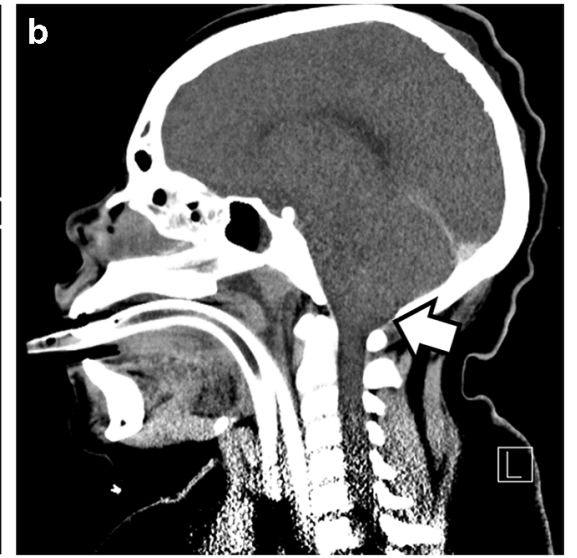

postmortem change, or antemortem anoxic injury. Scalp hematoma (thick arrow) was identified that was unsuspected clinically. b On this sagittal reformatted CT image, descent of the cerebellar tonsils (arrow) is another expected postmortem change that should not be assumed to represent antemortem pathology. CT did not detect right ventricular dysplasia with fatty infiltration of the right ventricular free wall identified at autopsy. CT confirmed the absence of fractures. The manner of death was "natural," and the cause of death was "anoxic encephalopathy following cardiac arrest" 


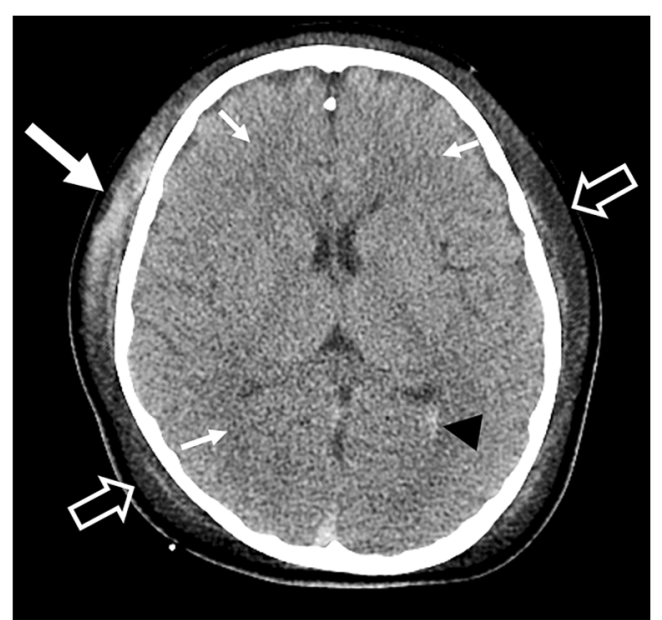

Fig. 8 Intraventricular hemorrhage in a 13-year-old boy who sustained extensive and fatal blunt force injuries in a motor vehicle accident the day before death. The interval between time of death and imaging was $3 \mathrm{~h}$. This axial CT image reveals intraventricular hemorrhage (black arrowhead) as well as scalp hematoma (large white arrow). Hemorrhage is not an expected postmortem finding and indicates injury that occurred prior to death. A subtle decrease in gray-white matter differentiation (small white arrows) might represent postmortem change, as may the bilateral scalp edema (black arrows). Postmortem CT was obtained following exploratory laparotomy and organ harvest later in the day. The study demonstrated multiple fractures in the axial and appendicular skeleton, intracranial injury as described, and postoperative changes in the chest, abdomen and pelvis. The autopsy report was unavailable

thromboembolism must be differentiated pathologically from coagulation that can occur in the right heart and pulmonary trunk because of very slow flow during a prolonged dying process [37]. Other expected cardiovascular postmortem changes include apparent thickening of the ventricular wall $[20,62]$ and aortic wall $[49,57,59]$ because of muscle contraction and mild right heart and vena cava dilatation from fluid stasis [49, 59] (Figs. 4 and 9).

Livor mortis is tissue discoloration also related to settling or stasis of blood and fluid within tissues and is seen as a gradual increase in attenuation in the more dependent portions of structures $[49,63]$. This finding is most noticeable in the subcutaneous fat (Fig. 10). Lung volumes decrease with an accompanying increase in interstitial fluid [20]. Increased attenuation from fluid in the dependent portions of the lungs should not be mistaken for pathology [20] (Fig. 11). Ground-glass opacity in the lungs is often seen postmortem and can easily mimic pneumonia [34]. Fluid can also accumulate postmortem within the pleural, pericardial and peritoneal cavities $[20,21]$ and might be difficult to differentiate from hydrops in fetuses and neonates [21]. Fluid within the airway and paranasal sinuses might represent postmortem change as well [59, 60, 64] (Fig. 12). The distribution of fluid within body cavities should be fairly uniform, and disproportionate accumulation in one space compared with others might indicate antemortem disease [59] (Fig. 9). Bowel distension is also
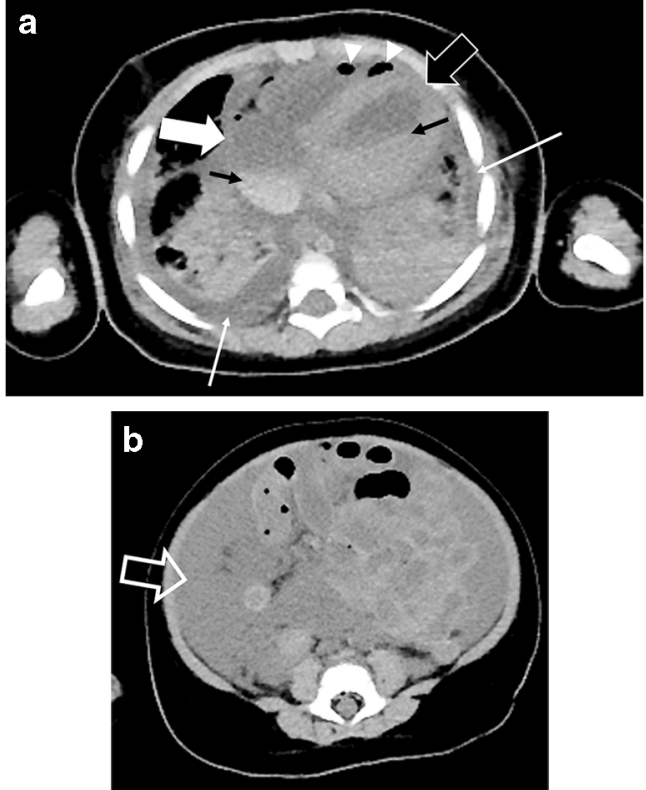

Fig. 9 Cardiovascular changes in a 4-month-old boy who was referred by the medical examiner for unexplained death. The interval between death and imaging is unknown because time of death was not known. a Gas in the right ventricle (arrowheads) might represent expected postmortem changes from putrefaction and cannot be presumed to represent antemortem pathology. In addition, a hematocrit effect is seen in the heart (thin black arrows) because of stasis of blood and settling of solid blood components. There is an asymmetrical amount of fluid in the pleural spaces (thin white arrows). Ventricular wall thickening (thick black arrow) from muscle contraction and right atrial enlargement (thick white arrow) are expected postmortem findings. b Axial CT image through the abdomen reveals a large amount of peritoneal fluid (arrow). While fluid accumulation is expected postmortem, it should be low volume, fairly symmetrical and uniform. In this case, the asymmetrical amount of fluid in the pleural spaces and large amount of intraperitoneal fluid suggest antemortem pathology and should prompt further investigation of the peritoneal cavity. CT confirmed the absence of trauma. Manner of death was deemed "natural." Cause of death was determined to be the result of "diffuse infection with CMV and myocarditis." CMV cytomegalovirus

a common postmortem finding, and the bowel might contain fluid, gas or both [20, 21, 57] (Fig. 13). Hyperdense fluid in the bowel or bowel wall on CT might represent hemorrhage [59]; however and investigation of this finding should be carried out at autopsy or with targeted sampling.

Gas formation is a common finding and can appear within cardiac chambers, vessels in the viscera and brain, and the bowel wall as well as the bowel lumen [20, 21, 49, 57, 60, 65] (Figs. 6, 9 and 14). Gas within cardiac chambers and vessels is also a known finding after cardiopulmonary resuscitation [49] and might not be related to putrefaction [65], although a causal relationship has not been demonstrated. Anterolateral rib fractures are associated with cardiac compressions [49] (Fig. 14). Inadvertent ventilation via the esophagus might add to the amount of gas within the bowel lumen [49] (Fig. 14). As time progresses, gas might be seen in additional compartments including the peritoneum and pleural 


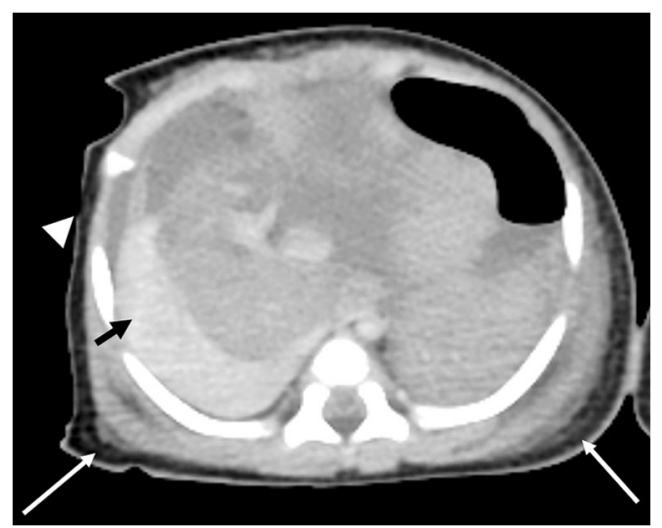

Fig. 10 Livor mortis. Axial CT image of a 6-month-old boy obtained $48 \mathrm{~h}$ after his unexplained death shows increased opacity in the dependent subcutaneous tissues (white arrows) secondary to livor mortis or homeostasis with settling of fluids and blood. This is an expected postmortem finding. High-attenuation fluid in the right pleural space (black arrow) was related to traumatic chest tube placement, known prior to death. The site of chest tube placement is visible as a defect in the skin (arrowhead). Early in our experience with postmortem imaging, the representatives from the medical examiner's office removed all monitoring leads, endotracheal tubes, drainage tubes and catheters. As we gained more experience, we asked that all catheters remain in place for the scan in order to document line positions and their relationship to trachea, stomach, tibias, etc. In this case, manner of death was determined to be "natural" and cause of death was signed out as "respiratory distress syndrome with contributing factor of traumatic intubation." CT excluded evidence of trauma but did not detect the cause of death because the extensive opacification of the lungs was indistinguishable from postmortem change

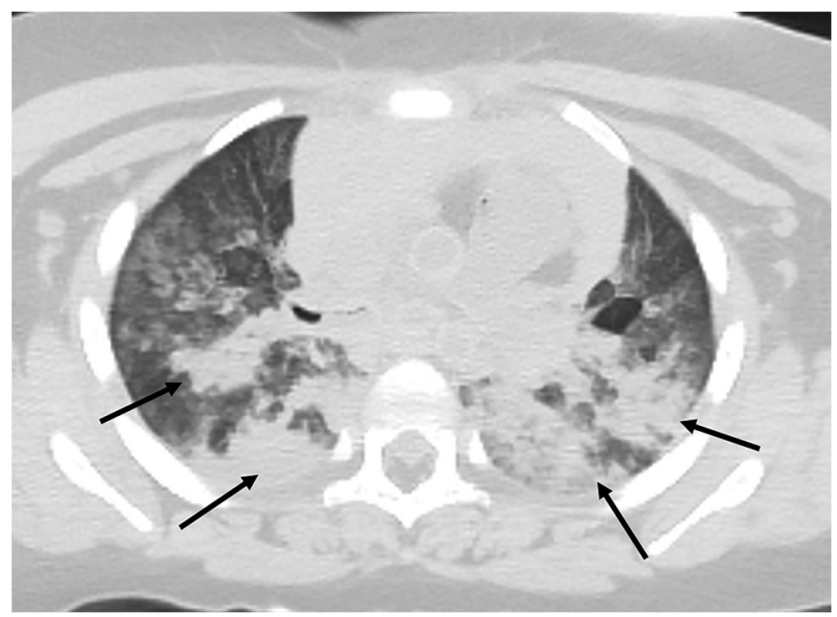

Fig. 11 Postmortem changes in the lungs in a 3-year-old boy who died following cardiac arrest. Imaging was performed more than $3 \mathrm{~h}$ after death. Axial CT image displayed with a lung window shows relatively symmetrical dependent opacities in the lungs (arrows). This does not necessarily represent antemortem pathology because pulmonary opacity, particularly when symmetrical and dependent, can be an expected postmortem change related to stasis. Autopsy found no evidence of aspiration or infection. No other abnormalities were found on CT, correlating with the autopsy findings. Manner of death was determined to be "natural" and cause of death was signed out as "idiopathic seizure disorder"

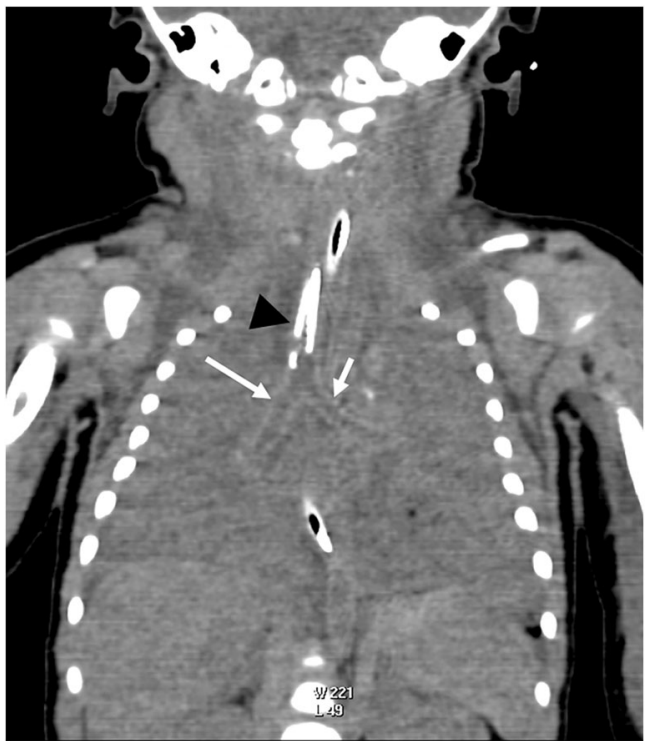

Fig. 12 Postmortem changes in the airways. This coronal reformatted CT image in a 4-week-old boy who was found unresponsive shows fluid in the trachea (arrows). Fluid in the airways and paranasal sinuses can be related to postmortem changes and should not be assumed to represent antemortem pathology. Autopsy found no respiratory system abnormalities. The interval between death and imaging was unknown. The endotracheal tube is well positioned in this case (arrowhead). CT found no evidence of trauma. Manner of death was determined to be "natural" and cause of death was signed out as "sudden unexplained infant death"

space [21]. Gas identified on postmortem imaging must be approached cautiously because it might not represent antemortem pathology.

Rigor mortis refers to stiffening of tissues 12-24 h after death and this can limit the technologist's ability to position the child for the postmortem imaging if scanning is performed during that time frame. Note also that rigor mortis might be difficult to differentiate from arthrogryposis in small fetuses [21].

\section{Our experience}

Hospital-based autopsies at our institution have decreased from roughly half of in-hospital deaths in 1997 to $18 \%$ in 2006. One of the authors with extensive experience in adult postmortem imaging and forensic radiology proposed our hospital institute a postmortem imaging program as a means for facilitating death investigation. Because of the existence of a formal statewide agreement, the "Memorandum of Understanding for the Multidisciplinary Response to Child Abuse and Neglect," the medical examiner was able to join with the hospital departments of medical imaging and pathology to develop a plan for performance of postmortem imaging examinations as well as to share diagnostic information within the patient confidentiality requirements of the Health 

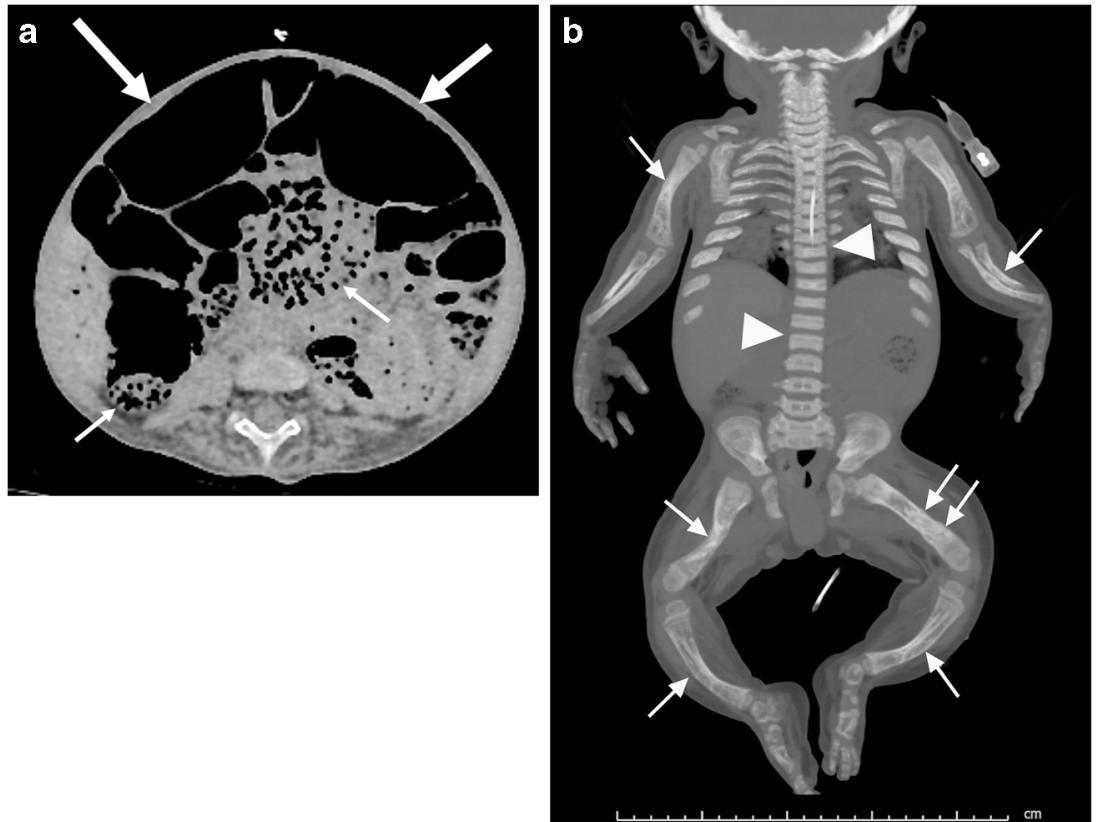

Fig. 13 Bowel distention in a 13-month-old girl with osteogenesis imperfecta who died after cardiac arrest. a Axial CT image shows gas both distending the bowel lumen (large arrows) and in the bowel wall (small arrows). In this case, there was at least a 3-h interval between the time of death and the CT scan. The intraluminal and intramural gas likely represents expected changes related to putrefaction. b Coronal maximumintensity projection $\mathrm{CT}$ image shows the healed fractures creating bowing

Insurance Portability and Accountability Act (HIPAA). The goal of the imaging program has been to provide information that might assist in determining cause of death; imaging is not intended to be a substitute for autopsy but to be an adjunct to the state medicolegal death investigations and the hospital's medical autopsies agreed to by families. In addition, postmortem imaging has looked at emergency medical interventions with the intent of providing clinicians with feedback on the intervention performed and equipment used. The focus has been on procedures used by first responders and our emergency medicine department. Because our institution does not provide obstetrical services, we have not performed fetal or newborn postmortem imaging.

Postmortem CT, and more recently MRI, protocols were developed through consultation with experienced adult postmortem imagers as well as review of the literature. We continue to work with our technologists to refine the protocols and extensive reconstructions. The scanning protocol is designed for maximal spatial resolution and minimal artifact because dose is not a concern. Our postmortem CT protocol is listed in Table 1. The entire body and head are scanned using 0.6-mm collimation to provide isotropic voxels for optimal 2-D and 3-D reconstruction. We perform separate reconstructions of the upper and lower segments of each extremity as well as of the head, spine, bony torso and bony pelvis. We have shared this protocol with other institutions in the state of

deformities of the extremities (single arrows). The double arrows indicate two foci of subtle periosteal reaction indicating incompletely healed fractures. Multiple vertebral compression deformities are identified (levels between the arrowheads). In this child, the manner of death could not be determined and the cause of death was attributed to "cardiorespiratory failure with contributing factors including chronic respiratory failure, ventilator dependent, and osteogenesis imperfecta"

Delaware with the goal of achieving a similar standard of practice statewide.

While most of our cases have been referred by the medical examiner, several cases have arisen from the emergency department and intensive care unit (Table 2). Cases that arise inhouse require consent from the parents. Working with the pathology department, we developed a consent form that allows parents to select postmortem imaging or noninvasive autopsy, including CT and MRI, autopsy, or both. Parents are advised that the combined investigation is thought to yield the most information.

Before the program was initiated, discussions with the technical staff occurred regarding appropriate timing of scans - whether they would occur during outpatient hours or later in the evenings. Postmortem CT is ideally performed as soon as possible after death to minimize decomposition changes, but we have performed exams after autopsy or even upon exhumed remains to provide skeletal evaluation. We have also worked with the technologists and hospital security to devise a protocol for transporting the deceased through the hospital to the medical imaging department while avoiding contact with patients and families. The technologists' input remains a valuable component of the ongoing process of refining our policies and imaging protocols.

To date, the state medical examiner has primarily used this service in our hospital for unexplained infant death when there 

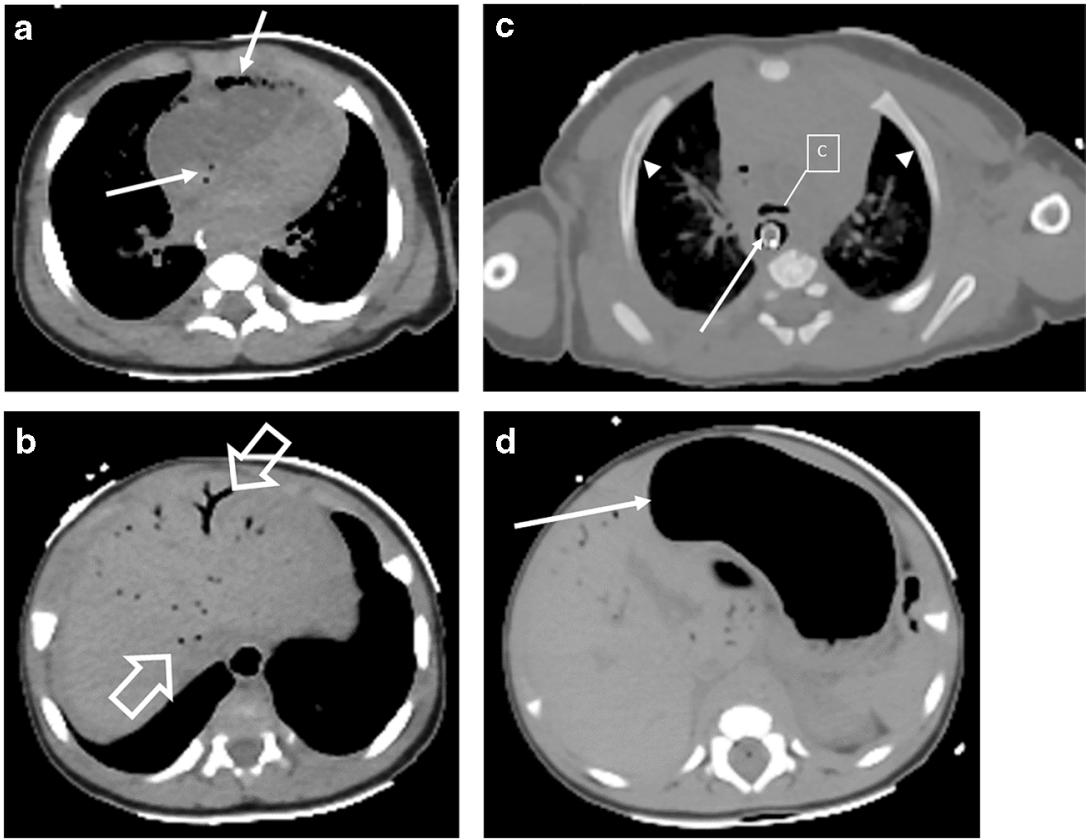

Fig. 14 Intracardiac gas on postmortem CT performed in an 8-week-old boy after attempted cardiopulmonary resuscitation (CPR). The interval between death and imaging was unknown; the boy arrived in the emergency department asystolic with fixed pupils. Time of death was unknown. a Intracardiac gas (arrows) has been described in deceased patients after CPR. b Gas can also be found in hepatic vessels (arrows) following attempted CPR. It is not known whether the findings are related to decomposition or the CPR itself. $\mathbf{c}$ Axial CT image with the bone window setting at the level of the 4 th ribs shows subtle bilateral buckle fractures of the

anterolateral ribs along their inner cortices (arrowheads). Fractures in this location are known to be associated with CPR, whereas posterior rib fractures are not. Inadvertent esophageal intubation (arrow) is identified ( $C$ - carina) on this axial CT image. $\mathbf{d}$ There is resulting pronounced gaseous dilatation of the stomach (arrow). In this child, manner of death could not be determined and cause of death was signed out as "sudden unexplained infant death." CT correctly identified the rib fractures but no other evidence of trauma

is a need to assess for non-accidental trauma. When abnormalities are identified that do not conform to expected postmortem changes, the imaging findings guide more in-depth gross and histopathological analysis (Figs. 2, 3, 4 and 5). Since initiating our program, we have collaborated with one of the authors who is an experienced pediatric radiologist and adult postmortem imager. We have found that even with prompt imaging after death there is a need to understand and recognize the decomposition process and related findings. Within the last 2 years we have begun to offer postmortem MRI in addition to postmortem CT to improve the detection of softtissue findings, particularly in the central nervous system, although we have performed very few MR studies. In our

Table 2 Demographics of children scanned at our hospital postmortem from 2011 to 2018

\begin{tabular}{ll}
\hline Number of patients & 78 \\
Age $<1$ year & 54 \\
Age $<5$ years & 68 \\
Referred by medical examiner & 70 \\
Referred by emergency department & 4 \\
Inpatient (intensive care unit) & 4 \\
Exhumed & 1 \\
Post-autopsy or organ harvest & 8 \\
\hline
\end{tabular}

experience postmortem CT has been effective in identifying pathology in areas not easily visualized by standard autopsy, particularly in the skeleton, as well as in assessing medical intervention (Fig. 15). We have found identification of occult fractures to be very useful. Equally as useful is being able to exclude $\mathrm{CT}$ evidence of fractures and intracranial hemorrhage. In cases of death by natural causes, we recognize the limitations of non-contrast postmortem $\mathrm{CT}$ and note that postmortem MRI is likely to provide opportunities to overcome these limitations [26].

\section{Challenges}

Postmortem CT in children is a very new field. Few radiologists are experienced in both pediatric/fetal imaging and postmortem/forensic imaging. Many radiologists have only limited experience with expected postmortem findings [14, 20]. At this point, collaboration with pathology or radiologists expert in differentiating ante- versus postmortem pathology might be needed because familiarity with expected postmortem changes is crucial for correct interpretation $[2,8,12,18$, $55,56]$, but this adds time to the interpretive process. Consultation with pathologists is necessary to verify imaging 


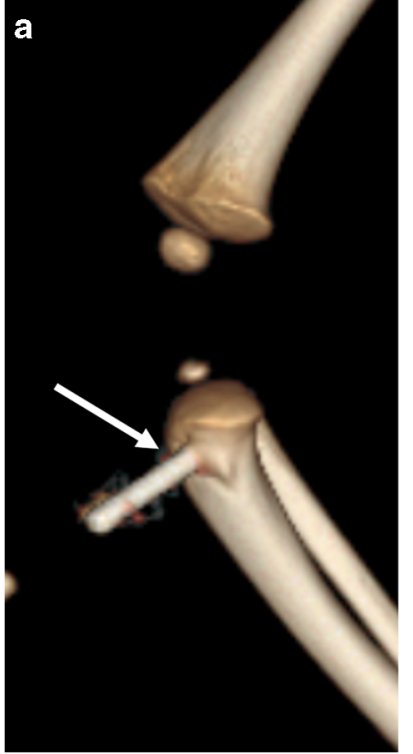

Fig. 15 Postmortem CT for assessing medical interventions. Volumerendered $\mathrm{CT}$ images of the knees in a 4-week-old boy with unexplained death. a The intraosseous cannula tip is well embedded in the proximal right tibial metaphysis (arrow). b However, in the left knee, the intraosseous cannula tip (arrow) is seen anterior to the tibial cortex rather than within the bone. Extensive subcutaneous edema and gas are

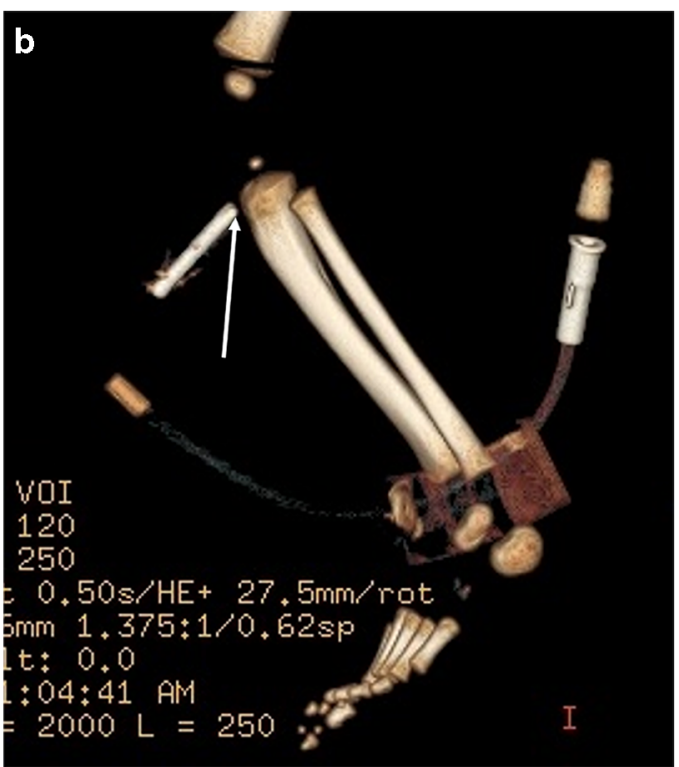

often seen in the setting of inadvertent extraosseous line placement. Linear bore holes might also be seen in the tibias from attempted unsuccessful line placements. Postmortem CT can be a useful tool in training and performance improvement for hospital staff and emergency medical technicians

hours reporting is impractical and can be difficult even during the day when there is a heavy routine caseload. Even transport issues can require some finesse, as discussed, involving managing the medical examiner's and clinical schedules and cadaver transport through patient areas. Finding available time on the MRI scanner and coordinating the timing of scanner availability with the state medical examiner have significantly limited our ability to utilize MR, despite the evidence indicating superior assessment of soft tissues with MRI.

Reimbursement for postmortem imaging is unclear $[10,11$, $18,44]$ and justification of a non-reimbursable study is problematic [14]. For investigations referred by the medical examiner, the judicial system covers the costs in some states. In the case of examinations performed in lieu of or in addition to autopsy, reimbursement is less clear. Third-party payers might not cover virtual autopsy, although some institutions might be able to bill for imaging of a stillbirth or fetus as a specimen study [44], similar to the charge generated for a breast biopsy specimen. Without a reliable plan for compensation, institutions might be reluctant to offer postmortem imaging or to develop the resources needed to provide a robust program. Our experience has been that the post-mortem CT cases we image are not reimbursed, meaning that the time spent on these cases by the radiologists during the workday reduces our apparent productivity in terms of billing. Alternatively, we read them after-hours. As a service to the community, our institution has been willing to absorb the scanner and technologist cost to perform these studies. 
Finally, the added value of postmortem CT is unproven. In adults, there is only a $50 \%$ rate of agreement for cause of death between postmortem imaging and autopsy, with a $32 \%$ major error rate for postmortem CT and a $43 \%$ error rate for postmortem MR $[9,19]$. In pediatric postmortem CT studies comparing concordance between postmortem CT and autopsy, agreement between the two procedures has been reported between $57 \%$ and $83 \%$ for all categories of findings [13, 33-35, 51]. Postmortem CT is superior to autopsy in detection of fractures [33], but evidence is limited that it is superior to radiographs in the setting of skeletal dysplasia [8, 12, 27]. Postmortem CT has been found to be better than radiography for rib fractures [47, 48]; however CT has been found to be more limited in the setting of natural death [33, 50, 52]. But when no cause of death is found by autopsy, concordance with CT has been shown to be very good $[34,51]$. The most common pitfalls reported in pediatric studies involving both CT and MRI are pulmonary disease, myocarditis and sepsis [34, 43]. Poor soft-tissue contrast is a significant limiting factor of postmortem CT $[8,41]$. Postmortem MRI has been shown to be superior to CT for evaluation of soft tissues [12, 41], most notably the central nervous system, heart and kidneys [8], particularly in fetuses and infants $[41,43]$. When postmortem CT is offered as an option to families in lieu of autopsy, it is important that the relative limitations of the study are explained $[8,9,68]$ with regard to both soft-tissue diagnosis and differentiation of postmortem changes from pathology.

\section{Conclusion}

Postmortem CT in children is an evolving application and its role compared to radiography, US and MRI has not been defined. Postmortem imaging in general might offer parents a noninvasive way to learn more about their child's disease for both bereavement and counseling [34, 68-70]. Imaging in addition to genetic analysis can provide a more complete assessment to guide counseling and planning for future pregnancies. The potential also exists to provide assistance to the judicial system with identifying cases of non-accidental trauma and providing evidence of that trauma $[6,13,33,35,47$, 51]. We have found postmortem CT promising in identifying or excluding non-accidental trauma in the setting of sudden infant death, but we are still compiling data to prove the added benefit of CT. Unlike physical remains that continue to decompose and that must be exhumed for further analysis, the electronic data stored from a postmortem CT can be revisited as many times as needed for as long as the data are archived as well as de-identified and shared for consultation and collaboration $[19,54]$. The imaging data are simple to reformat for courtroom display and can provide helpful visual confirmation for juries without the emotional distress of gross anatomical photography [19]. Findings related to support devices and surgical interventions might become the basis for feedback and instruction of first responders, emergency personnel and surgeons [36, 53, 55].

Continued work is needed to delineate reliable findings that can differentiate postmortem changes from antemortem pathology. Fractures, for example, strongly suggest antemortem trauma and can prompt focused attention to the appropriate area during autopsy along with correlation with history of cardiopulmonary resuscitation. Detection of antemortem brain edema and pulmonary airspace disease is more problematic regardless of imaging modality [34, 35, 43], something we have noted in our cases, as well. Systematic evaluation of postmortem cases must continue to search for findings that reliably differentiate between pre-mortem pathology and postmortem change. The number of papers describing imaging findings in specific conditions continues to increase, with extensive research having been completed in Europe [12], but there is still much to learn. Continued collaboration with pathology is required and findings on postmortem CT might help guide autopsy in some cases [67]. Pediatric radiologists are uniquely qualified to participate in perinatal and forensic imaging because we have training and experience in fetal/neonatal pathology as well as non-accidental trauma $[2,9,44]$. It is up to pediatric radiologists to demonstrate that pediatric postmortem imaging is reliable [17] and valuable enough to change the practice patterns of pathologists and medical examiners [16].

Building a postmortem imaging program from scratch requires collaborative planning for all aspects of the process including consent, transport, scanning technique and interpretation. To avoid disruption of clinical services and potentially upsetting both patients and technical staff, considerable cooperation is needed between services to orchestrate the transport and imaging of these cases. The importance of technologist training, cooperation and input cannot be overstated. Training for radiologists in the recognition of expected postmortem changes is imperative to avoid misinterpretation of expected findings as pathology. In addition, systematic corroboration of findings with clinicians, pathologists and the medical examiner, when appropriate, is needed to correlate antemortem clinical disease with postmortem findings and differentiate expected changes from true pathology. The lack of reimbursement is an additional hurdle that must be addressed. Particularly in the USA, agreement must be reached regarding utilization of radiologists and department resources with payment unlikely.

Will the virtual or imaging autopsy replace the physical autopsy? When postmortem imaging is offered along with other less-invasive techniques as an alternative to autopsy, the limitations in arriving at a cause of death must be explained. The roles of different imaging modalities for differing indications such as perinatal death versus unexplained infant death have yet to be fully established [3, 8, 43]. An ideal protocol might include MRI for central nervous system and soft tissues as well as CT if trauma or skeletal dysplasia is 
suspected or must be ruled out. Imaging can never fully supplant histopathological analysis. Performed properly, however, postmortem CT might provide a useful adjunct to traditional autopsy in select cases.

Acknowledgments Theresa Christensen was supported by the Nemours Departments of Biomedical Research (through the Nemours Summer Undergraduate Research Program) and Medical Imaging. Riley Curtin was supported by the Delaware Institutional Development Award (IDeA) Network of Biomedical Research Excellence program, with a grant from the National Institutes of Health National Institute of General Medical Sciences (grant \#P20 GM103446) and the state of Delaware, and by the Nemours Medical Imaging Department.

\section{Compliance with ethical standards}

Conflicts of interest None

\section{References}

1. Eriksson A, Gustafsson T, Hoistad M et al (2016) Diagnostic accuracy of postmortem imaging vs. autopsy - a systematic review. Eur J Radiol 89:249-269

2. Arthurs OJ, van Rijn RR, Sebire NJ (2014) Current status of paediatric post-mortem imaging: an ESPR questionnaire-based survey. Pediatr Radiol 44:244-251

3. Arthurs OJ, van Rijn RR (2015) Paediatric and perinatal postmortem imaging: mortui vivos docent. Pediatr Radiol 45:476-477

4. Baglivio M, Winklhofer S, Hatch GM et al (2013) The rise of forensic and post-mortem radiology - analysis of the literature between the year 2000 and 2011. J Forensic Radiol Imaging 1:3-9

5. Koopmanschap DH, Bayat AR, Kubat B et al (2016) The radiodensity of cerebrospinal fluid and vitreous humor as indicator of the time since death. Forensic Sci Med Pathol 12:248-256

6. Flach PM, Gascho D, Schweitzer W et al (2014) Imaging in forensic radiology: an illustrated guide for postmortem computed tomography technique and protocols. Forensic Sci Med Pathol 10:583-606

7. Cohen MC, Whitby EH, Fink MA et al (2015) Running a postmortem service - a business case and clinical experience. Pediatr Radiol 45:501-508

8. Arthurs OJ, Taylor AM, Sebire NJ (2015) Indications, advantages and limitations of perinatal postmortem imaging in clinical practice. Pediatr Radiol 45:491-500

9. Arthurs OJ, van Rijn RR, Taylor AM, Sebire NJ (2015) Paediatric and perinatal postmortem imaging: the need for a subspecialty approach. Pediatr Radiol 45:483-490

10. Karalis J, Denton E (2016) Forensic and post-mortem imaging in England: a national perspective. J Forensic Radiol Imaging 4:17-19

11. Arthurs OJ, van Rijn RR (2018) British Neuropathological Society and International Society for Forensic and Radiology Imaging expert consensus statment for post-mortem neurological imaging. J Forensic Radiol Imaging 13:3-4

12. Arthurs OJ, van Rijn RR, Whitby EH et al (2016) ESPR postmortem imaging task force where we begin. Pediatr Radiol 46:1363-1369

13. Shelmerdine SC, Gerrard CY, Rao P et al (2019) Joint European Society of Paediatric Radiology (ESPR) and International Society for Forensic Radiology nd imaging (ISFRI) guidelines: paediatric postmortem computed tomography imaging protocol. Pediatr Radiol 49:694-701
14. Chapman S (2015) What are the greatest challenges and/or barriers to applying postmortem imaging in paediatric radiology? Pediatr Radiol 45:478

15. Ruder TD (2015) What are the greatest challenges or barriers to applying post-mortem imaging in pediatric radiology? Pediatr Radiol 45:479

16. Hatch GM (2015) What are the greatest challenges or barriers to applying post-mortem imaging in pediatric radiology? Pediatr Radiol 45:480

17. Cain TM (2015) What are the greatest challenges or barriers to applying postmortem imaging in paediatric radiology? Pediatr Radiol 45:481

18. Alison M (2015) Postmortem imaging in paediatric radiology the French perspective. Pediatr Radiol 45:482

19. Gourincour G, Sarda-Quarello L, Laurent P-E et al (2015) The future of pediatric and perinatal postmortem imaging. Pediatr Radiol 45:5090516

20. Klein WM, Bosboom DG, Koopmanschap DH et al (2015) Normal pediatric postmortem CT appearances. Pediatr Radiol 45:517-526

21. Arthurs OJ, Barber JL, Taylor AM, Sebire NJ (2015) Normal perinatal and paediatric postmortem magnetic resonance imaging appearances. Pediatr Radiol 45:527-535

22. Calder AD, Offiah AC (2015) Foetal radiography for suspected skeletal dysplasia: technique, normal appearances, diagnositc approach. Pediatr Radiol 45:536-548

23. Taylor AM, Arthurs OJ, Sebire NJ (2015) Postmortem cardiac imaging in fetuses and children. Pediatr Radiol 45:549-555

24. Arthurs OJ, Chitty LS, Judge-Kronis L (2015) Postmortem magnetic resonance appearances of congenital high airway obstruction syndrome. Pediatr Radiol 45:556-561

25. Arthurs OJ (2015) ISFRI 2015: a turning point for paediatric PM imaging. J Forensic Radiol Imaging 3:180-181

26. Harcke HT (2010) The case for postmortem imaging. Pediatr Radiol 40:138-140

27. Arthurs OJ, Calder AD, Klein WM (2015) Is there still a role for fetal and perinatal post-mortem radiography? J Forensic Radiol Imaging 3:5-11

28. McNulty JP, Burke NP, Pelletier NA et al (2014) The impact of analogue and digital radiography for the identification of occult post-mortem fractures in neonates: a porcine model. J Forensic Radiol Imaging 2:20-24

29. deLange C, Vege A, Stake G (2007) Radiography after unexpected death in infants and children compared to autopsy. Pediatr Radiol 37:159-165

30. Peterson GF, Clark SC (2006) Forensic autopsy performance standards. Am J Forensic Med Pathol 27:200-225

31. Busardo FP, Frati P, Guglielmi G et al (2015) Postmortemcomputed tomography and postmortem-computed tomography-angiography: a focused update. Radiol Med 120:810-823

32. Donchin Y, Rivkind AI, Bar-Ziv J et al (1994) Utility of postmortem CT in trauma victims. J Trauma 37:552-555

33. Krentz BV, Alamo L, Grimm J et al (2016) Performance of postmortem CT compared to autopsy in children. Int J Legal Med 130: 1089-1099

34. Proisy M, Marchand AJ, Loget P et al (2013) Whole-body postmortem computed tomography compared with autopsy in the investigation of unexpected death in infants and children. Eur Radiol 23:1711-1719

35. Noda Y, Yoshimura K, Tsuji S et al (2013) Postmortem computed tomography imaging in the investigation of nontraumatic death in infants and children. Biomed Res Int 2013:327903

36. Zerlauth J, Doenz F, Dominguez A et al (2013) Surgical interventions with fatal outcome: utility of multi-phase postmortem CT angiography. Forensic Sci Int 225:32-41 
37. Ross SG, Bolliger SA, Ampanozi G et al (2014) Postmortem CT angiography: capabilites and limitations in traumatic and natural causes of death. Radiographics 34:830-846

38. Rutty GN, Morgan B, Germerott $\mathrm{T}$ et al (2016) Ventilated postmortem computed tomography - a historical review. J Forensic Radiol Imaging 4:35-42

39. Prodhomme O, Baud C, Saguintaah M et al (2015) Principles of fetal postmortem ultrasound: a personal review. J Forensic Radiol Imaging 3:12-15

40. Prodhomme O, Baud C, Saguintaah M et al (2015) Comparison of postmortem ultrasound and X-ray with autopsy in fetal death: retrospective study of 169 cases. J Forensic Radiol Imaging 3:120-130

41. Arthurs OJ, Guy A, Thayyil S et al (2016) Comparison of diagnostic performance for perinatal and paediatric post-mortem imaging: CT versus MRI. Eur Radiol 26:2327-2336

42. Eustler EP, Khanna G (2016) Whole-body magnetic resonance imaging in children: technique and clinical applications. Pediatr Radiol 46:858-872

43. Thayyil S, Sabire NJ, Chitty LS et al (2013) Post-mortem MRI versus conventional autopsy in fetuses and children: a prospective validation study. Lancet 382:223-233

44. Harty MP, Schmit P (2016) Pediatric image assisted autopsy: North American perspective. Presented at the sunrise session: post-mortem imaging. International Society for Pediatric Radiology, Chicago

45. Mazuchowski EL, Franco DM, Berran PJ, Harcke HT (2017) The virtual hydrostatic test. Am J Forensic Med Pathol 38:24-28

46. Barber JL, Sebire NJ, Chitty LS et al (2015) Lung aeration on postmortem magnetic resonance imaging is a useful marker of live versus stillbirth. Int J Legal Med 129:531-536

47. Hong TS, Reyes JA, Moineddin R et al (2011) Value of postmortem thoracic CT over radiography in imaging of rib fractures. Pediatr Radiol 41:736-748

48. Shelmerdine SC, Langan D, Hutchinson JC et al (2018) Chest radiographs versus $\mathrm{CT}$ for the detection of rib fractures in children (DRIFT): a diagnostic accuracy observational study. Lancet Child Adolesc Health 2:802-811

49. Offiah CE, Dean J (2016) Post-mortem CT and MRI: appropriate post-mortem imaging appearances and changes related to cardiopulmonary resuscitation. Br J Radiol 89:20150851

50. Oyake Y, Aoki T, Shiotani S et al (2006) Postmortem computed tomography for detecting causes of sudden death in infants and children: retrospective review of cases. Radiat Med 24:493-502

51. Sieswerda-Hoogendoorn T, Soerdjbalie-Maikoe V, deBakker H, van Rijn RR (2014) Postmortem CT compared to autopsy in children: concordance in a forensic setting. Int J Legal Med 128:957-965

52. van Rijn RR, Beek EJ, van de Putte EM et al (2017) The value of postmortem computed tomography in paediatric natural cause of death: a Dutch observational study. Pediatr Radiol 47:1514-1522

53. Jawad N, Brown K, Sebire NJ, Arthurs OJ (2016) Accuracy of paediatric intraosseous needle placement from post-mortem imaging. J Forensic Radiol Imaging 4:63-69

54. Ford JM, Decker SJ (2016) Computed tomography slice thickness and its effects on three-dimensional reconstruction of anatomical structures. J Forensic Radiol Imaging 4:43-46
55. Harty MP, Gould SW, Harcke HT et al (2015) The use of postmortem computed tomography (PMCT) in assessing pediatric deaths. Radiological Society of North America, Chicago

56. Smith A, Lattin G Jr, Berran P, Harcke HT (2012) Common and expected postmortem CT observations involving the brain: mimics of antemortem pathology. AJNR Am J Neuroradiol 33:1387-1391

57. Proisy M, Loget $\mathrm{P}$, Bouvet R et al (2015) Non-specific post-mortem modifications on whole-body post-mortem computed tomography in sudden unexpected death in infancy. J Forensic Radiol Imaging $3: 16-23$

58. Berger N, Ampanozi G, Scweitzer W et al (2015) Racking the brain: detection of cerebral edema on postmortem computed tomography compared with forensic autopsy. Eur J Radiol 84:643-651

59. Ishida M, Gonoi W, Okuma $\mathrm{H}$ et al (2015) Common postmortem computed tomography findings following atraumatic death: differentation between normal postmortem changes and pathologic lesions. Korean J Radiol 16:798-809

60. Sieswerda-Hoogendoorn T, Beenen L, van Rijn RR (2015) Normal cranial postmortem CT findings in children. Forensic Sci Int 246:43-49

61. Case ME (2014) Distinguishing accidental from inflicted head trauma at autopsy. Pediatr Radiol 44:S632-S640

62. Okuma H, Gonoi A, Ishida M et al (2013) Heart wall is thicker on postmortem computed tomography than on antemortem computed tomography: the first longitudinal study. PLoS One 8:e76026

63. Van Grinsven T, Lafebre SJ, Kubat B, Klein WM (2017) Postmortem changes in musculoskeletal and subcutaneous tissue. J Forensic Radiol Imaging 10:29-36

64. Biljardt S, Brummel A, Tijhuis R et al (2015) Post-mortem fluid stasis in the sinus, trachea and mainstem bronchi; a computed tomography study in adults and children. J Forensic Radiol Imaging 3:162-166

65. Barber JL, Kiho L, Sebire NJ, Arthurs OJ (2015) Interpretation of intravascular gas on postmortem CT in children. J Forensic Radiol Imaging 3:174-179

66. Miller Z (2013) Forensic radiology at the New York City office of the chief medical examiner (NYC OCME): current US forensic radiology practices and reflections on the future. J Forensic Radiol Imaging 1:84

67. Aalders M, Adolphi N, Daly B et al (2017) Research in forensic radiology and imaging; identifying the most important issues. $\mathrm{J}$ Forensic Radiol Imaging 8:1-8

68. Judge-Kronis L, Hutchinson J, Sebire NJ, Arthurs OJ (2016) Consent for paediatric and perinatal postmortem investigations: implications of less invasive autopsy. J Forensic Radiol Imaging 4:7-11

69. Berkovitz N, Tal S, Gottlieb P et al (2013) Introducing virtopsy into a country religiously opposed to autopsy. J Forensic Radiol Imaging 1:80

70. Vester M, van Rijn RR (2015) A knot in the gut: a paediatric postmortem CT diagnosis. J Forensic Radiol Imaging 3:238-240

Publisher's note Springer Nature remains neutral with regard to jurisdictional claims in published maps and institutional affiliations. 